\title{
Attenuation of Aircraft Flexible Modes during Maneuvering Flight
}

\author{
Fabio A. de Almeida* \\ IAE, Instituto de Aeronáutica e Espaço, 12228-904 São José dos Campos, Brazil \\ Bruno Giordano de Oliveira Silva ${ }^{\dagger}$ \\ DLR, German Aerospace Center, 38108 Braunschweig, Germany
}

This work presents a new method to deal with aeroelastic phenomena which interfere in the motion and dynamics of aircraft. By suppressing the accelerations of flexible aircraft modes, the related structural displacements and velocities are diminished, providing reduction of structural loads and improving passengers comfort, since undesirable high-frequency vibration content is practically eliminated. The proposed attenuation is achieved by means of a control technique denominated constrained linear quadratic control, which basically is the well-known linear quadratic regulator with addition of inequality constraints on the state and input variables. An aeroservoelastic model is presented where flexible and rigid body dynamics are integrated in a single set of equations. Simulation results of the complete closed-loop system, with limitations imposed over the modal accelerations, demonstrate the possibility to maneuver the flexible aircraft with reduced flexible displacements and velocities.

\section{Nomenclature}

$\begin{array}{ll}A, B & \text { continuous-time state-space matrices } \\ a_{y} & \text { lateral acceleration, } \mathrm{m} / \mathrm{s}^{2} \\ b & \text { aircraft wingspan, m } \\ c & \text { mean aerodynamic chord, m } \\ C_{c} & \text { output constraint matrix } \\ C_{X}, C_{Y}, C_{Z} & \text { dimensionless specific forces in body-axes, adm } \\ d_{m a x}, d_{m i n} & \text { bounds over disturbance vector } \\ D O F & \text { degrees-of-freedom } \\ E & \text { observation matrix } \\ E_{a} & \text { augmented observation matrix } \\ H & \text { tracking matrix } \\ I & \text { identity matrix } \\ J & \text { cost function } \\ K_{d} & \text { feedback gain matrix } \\ L, L_{d}, L_{x} & \text { observer gain matrices } \\ \overline{L_{d}}, \bar{H} & \text { auxiliary observer matrices } \\ \mathcal{L}, \mathcal{M}, \mathcal{N} & \text { aerodynamic moments along body-fixed axes, N.m } \\ \mathcal{L}_{T}, \mathcal{M}_{T}, \mathcal{N}_{T} & \text { propulsive moments along body-fixed axes, N.m } \\ L Q R & \text { linear quadratic regulator } \\ m & \text { mass, kg }\end{array}$

${ }^{*}$ Chief Researcher, Aeronautical Systems Division (ASA), Pça Marechal Eduardo Gomes 50 - Brazil, e-mail: almeidafaa@iae.cta.br, AIAA Senior Member.

${ }^{\dagger}$ Guest Scientist, Institute of Flight Systems, Lilienthalplatz 7 on leave from DCTA/IPEV - Brazil, e-mail: epd-t@ipev.cta.br, AIAA Member. 


\begin{tabular}{|c|c|}
\hline$M P C$ & model predictive control \\
\hline$O_{\infty}$ & invariant set \\
\hline$P$ & terminal weighting matrix \\
\hline$p, q, r$ & aircraft angular rates, $\mathrm{rad} / \mathrm{s}$ \\
\hline$Q, R$ & continuous weighting matrices \\
\hline$Q_{d}, W_{d}, R_{d}$ & discrete weighting matrices \\
\hline$Q_{s s}, R_{s s}$ & constrained target calculator weighting matrices \\
\hline$Q P$ & quadratic programming \\
\hline $\mathcal{Q}$ & generalized aerodynamic load, $\mathrm{N}$ \\
\hline$r_{c}$ & command (demand) vector \\
\hline$r_{s s}$ & statically admissible command vector \\
\hline$S$ & wing area, $\mathrm{m}^{2}$ \\
\hline$T$ & net thrust, $\mathrm{N}$ \\
\hline$T_{s}$ & sampling time, $\mathrm{s}$ \\
\hline$t$ & time, $\mathrm{s}$ \\
\hline$T_{d}$ & rotation matrix \\
\hline$u_{\max }, u_{\min }$ & bounds over control vector \\
\hline$u, v, w$ & aircraft true airspeed components in body-fixed axes, $\mathrm{m} / \mathrm{s}$ \\
\hline $\mathbf{U}_{s s_{k}}$ & feasible steady-state control set \\
\hline $\mathbf{X}, \mathbf{U}$ & admissible state and control sets \\
\hline$x, u$ & state and control vectors \\
\hline$x_{a}$ & augmented state vector \\
\hline$x_{0}$ & initial state vector \\
\hline$\hat{x}, \hat{d}$ & estimated state and disturbance vectors \\
\hline$x_{\max }, x_{\min }$ & bounds over state vector \\
\hline$x_{s s_{\max }}, x_{s s_{\min }}$ & bounds over state vector at steady-state \\
\hline$x_{s s}, u_{s s}$ & state and control vectors at steady-state \\
\hline$\tilde{x}, \tilde{u}$ & tracking state and control vectors \\
\hline$X, Y, Z$ & aerodynamic forces along body-fixed axes, $\mathrm{N}$ \\
\hline$X_{T}, Y_{T}, Z_{T}$ & propulsive forces along body-fixed axes, $\mathrm{N}$ \\
\hline$y$ & observed vector \\
\hline$z$ & controlled output vector \\
\hline$z_{s s}$ & feasible command vector \\
\hline$\alpha, \beta$ & angles of attack and sideslip, rad \\
\hline$\delta_{e}, \delta_{a}, \delta_{r}$ & elevator, aileron, and rudder deflections, rad \\
\hline$\delta_{e_{c}}, \delta_{a_{c}}, \delta_{r_{c}}$ & elevator, aileron, and rudder commands, rad \\
\hline$\delta_{t}$ & deviation over trimmed thrust, $\mathrm{N}$ \\
\hline$\delta_{t_{c}}$ & thrust command, $\mathrm{N}$ \\
\hline$\eta, \dot{\eta}, \ddot{\eta}$ & generalized modal displacement, velocity, and acceleration, adm \\
\hline$\gamma$ & flight path angle, rad \\
\hline$\mu$ & generalized modal mass, kg.m ${ }^{2}$ \\
\hline$\omega$ & generalized modal natural frequency, rad/s \\
\hline $\bar{\Phi}$ & modal shape vector \\
\hline$\phi, \theta, \psi$ & Euler angles, rad \\
\hline$\Phi, \Gamma, \Gamma_{d}$ & discrete-time state-space matrices \\
\hline$\Phi_{a}, \Gamma_{a}$ & augmented discrete state-space matrices \\
\hline $\bar{\Phi}$ & auxiliary discrete state-space matrix \\
\hline$\dot{\psi}$ & turn rate, $\mathrm{deg} / \mathrm{s}$ \\
\hline$\sigma$ & slice operator \\
\hline$\zeta$ & generalized modal damping ratio, adm \\
\hline
\end{tabular}

\section{Introduction}

The interaction of aircraft flexible modes and rigid-body dynamics represents an important topic in aeronautics because, from a certain point of view, flexibility and related phenomena interfere in the classical 
air vehicle motion. Starting some decades ago with the understanding of how structural vibrations are excited by turbulent air and gusts, and arriving recently at very flexible aircraft, which slow dynamics can not be characterized only by simple rigid-body modes, this complex relationship poses several challenges during the design phase. Structural dynamic modes, if neglected, may compromise the vehicle fatigue life by increasing fuselage and wing loads, decrease passenger comfort and handling qualities, or even put at risk the closed-loop performance and stability of control systems.

Thus, it seems clear that the first step to deal with such phenomena is the adequate characterization of flexible vehicles dynamics, formulating a model capable of providing answers and solutions for the aforementioned problems. From the flight mechanics point-of-view, one of the first approaches ${ }^{1,2}$ was to consider that the internal structural motion is damped much faster than the rigid-body translational and rotational motions, which allow one to assume that the flexibility effects can be accounted by a proper modification on the rigid-body stability and control derivatives. This approach does not take into account the accelerations and transient forces caused by structural motion.

In the noteworthy work of Milne, ${ }^{3}$ the equations of motion of the deformable airplane are derived analytically, assuming a general nonlinear rigid-body motion and small structural displacements. Airplane structure is idealized as a beam in longitudinal bending and aerodynamic forces are obtained by a superposition of first-order effects about an equilibrium condition of a slender wing. The issue of choosing a suitable axis system is also addressed and the mean axes reference system is adopted in order to simplify the equations of motion, decoupling inertially the rigid and flexible degrees of freedom.

Assuming validity of linear elastic theory, availability of a set of orthogonal modes, collinearity of structural displacements and rates and neglecting the inertia tensor changes due to deformation, Waszak and Schmidt ${ }^{4}$ derive the equations of motion of the flexible aircraft relative to mean axes by application of Lagrange's equation. The Principle of Virtual Work is used to derive the generalized forces. This development results in a set of six scalar first-order equations for the rigid-body motion relative to mean axes reference system that are formally equivalent to the conventional equations of motion from the Flight Mechanics and one second-order differential equation in terms of modal displacements for each structural mode retained in the truncated model. Through a quasi-steady strip aerodynamic theory, the aerodynamic forces are obtained analytically, in terms of generalized stability and control derivatives, which provide the coupling between rigid and flexible states.

A contemporary work from Buttrill et al. ${ }^{5}$ also developed the equations of motion of the flexible aircraft using Lagrangian mechanics. In that work, the changes in the inertia tensor due to elastic displacements are accounted for and the assumption of collinearity between structural displacements and velocities is not adopted. As a result, the equations for rigid-body rotations and for structural motion are considerably more involved and coupled through nonlinear inertial terms. The aerodynamic forces due to elastic modes were obtained through a rational function approximation of frequency responses derived from a doublet-lattice model. The comparison on the responses obtained through inertially coupled and decoupled simulations indicated that the effects of coupling on rigid-body responses were negligible, whereas some elastic modes were significantly affected by inertial coupling, especially in maneuvers containing high roll rates, with magnitude comparable to the natural frequency of elastic modes. A work by Waszak et al. ${ }^{6}$ compares both approaches and highlights their consistency, stating that the inertially uncoupled equations of motion from Ref. [4] can be derived immediately from the coupled equations obtained in Ref. [5] simply by applying the proper assumptions.

In the last decade, different research groups have made efforts in the search of high-fidelity flexible aircraft time-domain simulation models. ${ }^{7-10}$ The structural dynamics is usually modeled through high-resolution Finite Element and the aerodynamic forces are obtained through CFD methods. These simulations have also as a common characteristic a very high computational demand, and the accuracy of the integrated model is not yet validated by comparison with real flight test data.

Flight tests have been performed in order to identify suitable models of the dynamics of aircraft considered as a flexible body. An investigation performed at Airbus ${ }^{11}$ has obtained a black box model for a large transport aircraft, using an eigensystem realization algorithm (ERA), in order to provide data for control law synthesis aiming at improvement of passenger comfort. Another work used the frequency-response method to identify a linear state-space model for the lateral-directional dynamics of a Boeing transport aircraft, incorporating the first anti-symmetric wing and fuselage bending modes. ${ }^{12}$

Recently, a work by de Oliveira Silva ${ }^{13}$ demonstrated the feasibility of using the output-error method in time domain to obtain a model for an aircraft that incorporates the coupled dynamics of the rigid body 
and structural motion, by considering a model comprising the longitudinal motion augmented by the first symmetric wing bending mode. An extension of the results is presented in Ref. [14], where a model containing three symmetric and two anti-symmetric structural modes is identified and validated. Both Refs. [13] and [14] use a model based on the one developed at Ref. [4], where the coupling between rigid body and structural motion is obtained through generalized derivatives, which seems to be a natural extension of the well-established approach adopted in the system identification of rigid body aerodynamic models.

The correct definition of an unified flight mechanics and aeroelastic model opens a wide variety of solutions to problems related with flexibility. Shearer and Cesnik ${ }^{15}$ presents a comprehensive review of control applications for aeroelastic vehicles and also proposes a trajectory control system for very flexible aircraft, where simulations show clearly the effect of flexibility in the closed-loop motion of the aircraft. A robust $H_{\infty}$ control for pitch rate tracking of a flexible aircraft is proposed by Silvestre and Paglione, ${ }^{16}$ demonstrating adequate performance in the presence of atmospheric turbulence. Wildschek et al. ${ }^{17,18}$ introduces an adaptive feedforward controller to provide wing load alleviation, identifying on-line the frequency content of important flexible modes based on accelerometers placed along the fuselage and wing. Recently, Haghighat, Liu and Martins applied nonlinear control techniques such as Model Predictive Control, ${ }^{19}$ Robust Linear Matrix Inequality ${ }^{20}$ and Robust Mixed-Norm Multi-Objective, ${ }^{21}$ considering an aeroservoelastic model with integrated rigid-body dynamics and flexible dynamics.

The research advances in the field of flexible aircraft, and its control, indicates some possibilities to go further. But, despite the availability of integrated aeroservoelastic models and advanced control techniques, the vast majority of the proposed solutions are capable of dealing only with structural vibration suppression after turbulence encounter. Flexible phenomena induced by maneuvering have been practically not considered. Only recently, Frost et al. ${ }^{22}$ presented a linear quadratic tracker with control allocation and explicit consideration of maximum wing structural loads as constraints in the optimal constrained problem, assuming an over-actuated large transport aircraft.

Therefore, this work proposed a novel approach to deal with aeroelastic phenomena, establishing bounds over the flexible modes accelerations. Doing so, the structural displacements and velocities are suppressed, reducing structural loads and improving passenger comfort. High-frequency vibrations are also strongly attenuated. Those benefits are achieved by means of a LQR-type technique, denominated Constrained Linear Quadratic Tracker (CLQT), ${ }^{23}$ with intuitive tuning parameters. The controller tracks commanded variables and, at the same time, limits the flexible modal accelerations. The synthesis of the controller practically does not account for flexibility in the gains computation, avoiding feedback of structural dynamics in the flight control system. The proposed control system is applied to the B1 Bomber model of Waszak and Schmidt. ${ }^{4}$ Longitudinal and lateral abrupt maneuvers are simulated, demonstrating notable performance and stability.

\section{Aeroservoelastic Model}

The model developed in this section is based on the work of Waszac and Schmidt. ${ }^{4}$ The derivation of the equations of motion and the inertial decoupling between the rigid body and structural dynamics is obtained through the use of the mean axes reference system and the following assumptions:

- Structural deformation is assumed to be small and described by a set of eigenmodes;

- Moments and products of inertia do not vary with deformation;

- Aerodynamic forces are modeled by quasi-steady strip theory; and

- Deformation and deformation rates are collinear.

The second assumption allows one to write the structural displacement in a point of the aircraft's structure as an infinite sum of contributions from its normal modes:

$$
\vec{d}(x, y, z, t)=\sum_{i=1}^{\infty} \vec{\Phi}_{i}(x, y, z) \eta_{i}(t)
$$

In Eq. (1) the vector $\vec{\Phi}_{i}$ represents the shape of the flexible modes and has dimension of length, whereas $\eta_{i}$ is a non-dimensional scalar function of the time and represents the generalized displacement of the respective mode. 
For real applications in simulation, the sum in Eq. (1) must be truncated at some point, which means that some normal modes should be retained and the remaining ones should be disregarded. If the interest lays on the influence of the flexibility on the flight mechanics, the most straightforward way to make this selection is to retain the modes with lowest natural frequencies, which have greater probability of influencing the low-frequency rigid-body modes dynamically or be influenced by them.

From the kinetic and potential energy expressions (including the energy terms due to structural deformation), the application of Lagrange's equation leads to the set of equations represented by (2):

$$
\begin{gathered}
\dot{u}-r v+q w+g \sin \theta=\frac{X+X_{T}}{m} \\
\dot{v}-p w+r u-g \sin \phi \cos \theta=\frac{Y+Y_{T}}{m} \\
\dot{w}-q u+p v-g \cos \phi \cos \theta=\frac{Z+Z_{T}}{m} \\
I_{x x} \dot{p}-\left(I_{x y} \dot{q}+I_{x z} \dot{r}\right)+\left(I_{z z}-I_{y y}\right) q r+\left(I_{x y} r-I_{x z} q\right) p+\left(r^{2}-q^{2}\right) I_{y z}=\mathcal{L}+\mathcal{L}_{T} \\
I_{y y} \dot{q}-\left(I_{x y} \dot{p}+I_{y z} \dot{r}\right)+\left(I_{x x}-I_{z z}\right) p r+\left(I_{x y} p-I_{y x} r\right) q+\left(p^{2}-r^{2}\right) I_{x z}=\mathcal{M}+\mathcal{M}_{T} \\
I_{z z} \dot{r}-\left(I_{x z} \dot{p}+I_{y z} \dot{q}\right)+\left(I_{y y}-I_{x x}\right) p q+\left(I_{x z} q-I_{y z} p\right) r+\left(q^{2}-p^{2}\right) I_{x y}=\mathcal{N}+\mathcal{N}_{T} \\
\ddot{\eta}_{i}+2 \zeta_{i} \omega_{i} \dot{\eta}_{i}+\omega_{i}^{2} \eta_{i}=\frac{\mathcal{Q}_{i}}{m_{i}}
\end{gathered}
$$

The first six equations in (2) are written with regard to the mean axes reference system and are formally equivalent to the classical equations for rigid body dynamics, whereas the last expression is equivalent to the classical flutter equation. Due to the assumption of constant inertia and use of mean axes system, the rigid body and the structural motion are coupled solely through the aerodynamic forces at the right hand side.

In the right-hand side of Eq. (2), $X, Y$ and $Z$ are the external forces acting in the respective axes. $\mathcal{L}$, $\mathcal{M}$, and $\mathcal{N}$ are the external moments. The subscript $\mathrm{T}$ refers to the propulsive terms. The aircraft mass and moments and products of inertia are given by $m, I_{x x}, I_{y y}, I_{z z}, I_{x y}, I_{x z}$, and $I_{y z}$. Here it is assumed that the inertia tensor does not vary with structural deformation.

The generalized force acting on the i-th structural mode is represented by $Q_{i}$ and has aerodynamic source. The modal mass, in-vacuum natural frequency, and damping ratio are, respectively, $m_{i}, \omega_{i}$, and $\zeta_{i}$.

\section{A. Structural Dynamics}

In the present work, the simulated aircraft corresponds to the B-1 model from Waszak et al. Hence, The structural dynamics is represented by two normal modes - one symmetric and one anti-symmetric. ${ }^{16,24}$ The modal characteristics are presented in Table 1 and a graphical representation of these structural modes is depicted in Fig. 1. The symmetric mode is characterized by wing and fuselage bending, whereas the anti-symmetric mode is mainly characterized by torsion of wing and fuselage.

Table 1. Modal parameters

\begin{tabular}{ccc} 
Parameter & Mode 1 symmetric & Mode 2 anti-symmetric \\
\hline Modal mass $\left(\mathrm{kg} \cdot \mathrm{m}^{2}\right)$ & $2.18 \times 10^{2}$ & $3.93 \times 10^{4}$ \\
Natural frequency $(\mathrm{rad} / \mathrm{s})$ & 12.57 & 14.07 \\
Damping & 0.02 & 0.02
\end{tabular}

\section{B. Aerodynamics}

Waszak and Schmidt ${ }^{4}$ used the strip theory and assumption of quasi-steady aerodynamics in order to derive the expressions for the aerodynamic coefficients acting on the rigid body degrees-of-freedom, as well as the aerodynamic coefficients for the generalized loads. The main result of this approach was the formulation of a set of aerodynamic coefficients that depend linearly on the modal displacements and their rate in time. 

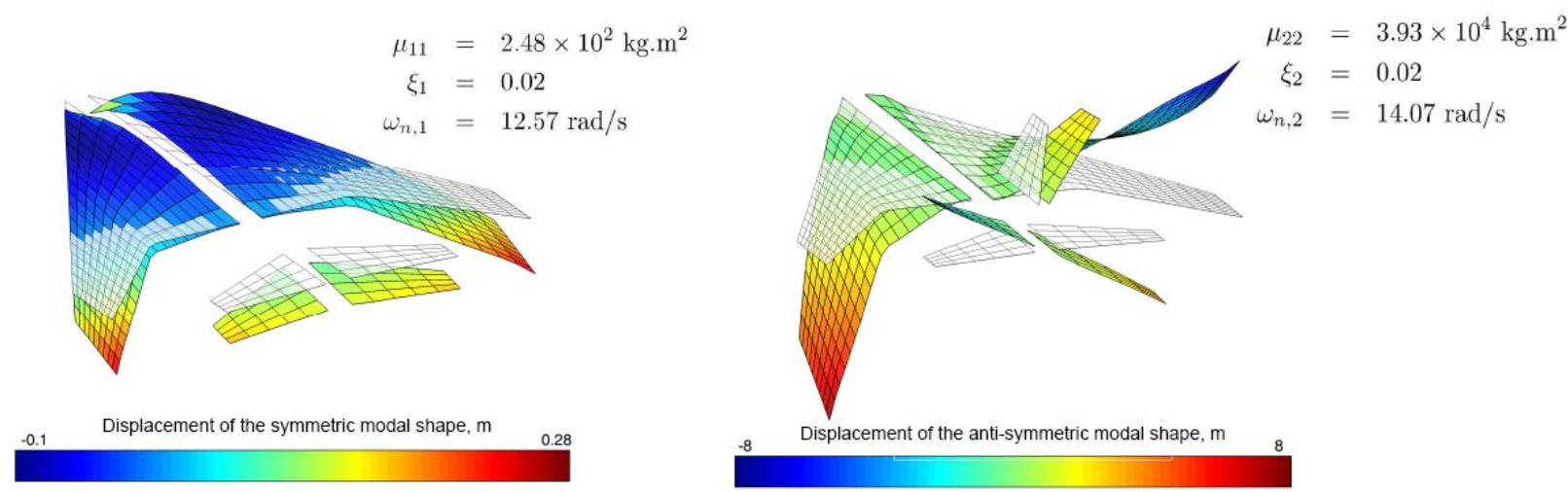

Figure 1. Eigenforms of the (a) symmetric and (b) anti-symmetric structural modes (adapted from Silvestre and Paglione ${ }^{16}$ )

Due to symmetry properties, the lateral-directional coefficients are influenced only by the anti-symmetric structural motion, whereas the longitudinal coefficients are influenced only by the symmetric motion.

So, the expressions for the aerodynamic coefficients acting on the rigid body degrees-of-freedom can be written as:

$$
\begin{aligned}
C_{X} & =C_{X_{0}}+C_{X_{\alpha}} \alpha+C_{X_{\delta_{e}}} \delta_{e}+C_{X_{q}} \frac{q \bar{c}}{2 V}+C_{X_{\eta_{1}}} \eta_{1}+C_{X_{\dot{\eta}_{1}}} \frac{\dot{\eta}_{1} \bar{c}}{2 V} \\
C_{Z} & =C_{Z_{0}}+C_{Z_{\alpha}} \alpha+C_{Z_{\delta_{e}}} \delta_{e}+C_{Z_{q}} \frac{q \bar{c}}{2 V}+C_{Z_{\eta_{1}}} \eta_{1}+C_{Z_{\dot{\eta}_{1}}} \frac{\dot{\eta}_{1} \bar{c}}{2 V} \\
C_{\mathcal{M}} & =C_{\mathcal{M}_{0}}+C_{\mathcal{M}_{\alpha}} \alpha+C_{\mathcal{M}_{\delta_{e}}} \delta_{e}+C_{\mathcal{M}_{q}} \frac{q \bar{c}}{2 V}+C_{\mathcal{M}_{\dot{\alpha}}} \frac{\dot{\alpha} \bar{c}}{2 V}+C_{\mathcal{M}_{\eta_{1}}} \eta_{1}+C_{\mathcal{M}_{\dot{\eta}_{1}}} \frac{\dot{\eta}_{1} \bar{c}}{2 V} \\
C_{Y} & =C_{Y_{\beta}}+C_{Y_{\delta_{a}}} \delta_{a}+C_{Y_{\delta_{r}}} \delta_{r}+C_{Y_{\eta_{2}}} \eta_{2}+C_{Y_{\dot{\eta}_{2}}} \frac{\dot{\eta}_{2} \bar{c}}{2 V} \\
C_{\mathcal{L}} & =C_{\mathcal{L}_{\beta}}+C_{\mathcal{L}_{\delta_{a}}} \delta_{a}+C_{\mathcal{L}_{\delta_{r}}} \delta_{r}+C_{\mathcal{L}_{p}} \frac{p b}{2 V}+C_{\mathcal{L}_{r}} \frac{r b}{2 V}+C_{\mathcal{L}_{\eta_{2}}} \eta_{2}+C_{\mathcal{L}_{\dot{\eta}_{2}}} \frac{\dot{\eta}_{2} \bar{c}}{2 V} \\
C_{\mathcal{N}} & =C_{\mathcal{N}_{\beta}}+C_{\mathcal{N}_{\delta_{a}}} \delta_{a}+C_{\mathcal{N}_{\delta_{r}}} \delta_{r}+C_{\mathcal{N}_{p}} \frac{p b}{2 V}+C_{\mathcal{N}_{r}} \frac{r b}{2 V}+C_{\mathcal{N}_{\eta_{2}}} \eta_{2}+C_{\mathcal{N}_{\dot{\eta}_{2}}} \frac{\dot{\eta}_{2} \bar{c}}{2 V}
\end{aligned}
$$

where $V$ is the true airspeed calculated as

$$
V=\sqrt{u^{2}+v^{2}+w^{2}}
$$

Similarly, the expressions for the generalized loads acting on the flexible modes are given by

$$
\begin{aligned}
& C_{1}=C_{0}^{\eta_{1}}+C_{\alpha}^{\eta_{1}} \alpha+C_{\delta_{e}}^{\eta_{1}} \delta_{e}+C_{q}^{\eta_{1}} \frac{q \bar{c}}{2 V}+C_{\eta_{1}}^{\eta_{1}} \eta_{1}+C_{\eta_{1}}^{\eta_{1}} \frac{\dot{\eta}_{1} \bar{c}}{2 V} \\
& C_{2}=C_{\beta}^{\eta_{2}} \beta+C_{\delta_{a}}^{\eta_{2}} \delta_{a}+C_{\delta_{r}}^{\eta_{2}} \delta_{r}+C_{p}^{\eta_{2}} \frac{p b}{2 V}+C_{r}^{\eta_{2}} \frac{r b}{2 V}+C_{\eta_{2}}^{\eta_{2}} \eta_{2}+C_{\eta_{2}}^{\eta_{2}} \frac{\dot{\eta}_{2} \bar{c}}{2 V}
\end{aligned}
$$

The angles of attack $\alpha$ and sideslip $\beta$ are obtained from the states of Eq. (2) accounting for the variable wind effects through

$$
\begin{aligned}
& \alpha=\arctan \left(\frac{w-w_{w}}{u-u_{w}}\right) \\
& \beta=\arcsin \left(\frac{v-v_{w}}{\sqrt{\left(u-u_{w}\right)^{2}+\left(v-v_{w}\right)^{2}+\left(w-w_{w}\right)^{2}}}\right)
\end{aligned}
$$


The rigid body aerodynamic coefficients are converted to forces and moments through the relations

$$
\begin{aligned}
X & =\bar{q} S C_{X} & & \mathcal{L}=\bar{q} S b C_{\mathcal{L}} \\
Y & =\bar{q} S C_{Y} & & \mathcal{M}=\bar{q} S \bar{c} C_{\mathcal{M}} \\
Z & =\bar{q} S C_{Z} & & \mathcal{N}=\bar{q} S b C_{\mathcal{N}}
\end{aligned}
$$

In a similar way, the generalized loads are obtained from the respective coefficients through

$$
\begin{aligned}
& \mathcal{Q}_{1}=\bar{q} S \bar{c} C_{1} \\
& \mathcal{Q}_{2}=\bar{q} S \bar{c} C_{2}
\end{aligned}
$$

The stability and control derivatives from Eqs. (3) and (5) used in this work are provided by Silvestre and Paglione. ${ }^{16}$

\section{Propulsion}

As the propulsion model is not available, and for the sake of simplicity, it is assumed here that the propulsion force acts on the center of gravity and is directed towards the $\mathrm{x}$ axis.

A maximum thrust of $259600 \mathrm{~N}$ is assumed, varying with the atmospheric density through the law:

$$
T_{c}=\delta_{t_{c}} T_{\max }\left(\frac{\rho}{\rho_{0}}\right)^{0.7}
$$

A first order delay of $0.33 \mathrm{~s}$ is assumed to exist between the actual and commanded thrust.

\section{Constrained Linear Quadratic Tracker for Attenuation of Flexible Modes}

This section presents the steps to synthesize the constrained linear quadratic tracker. ${ }^{23}$ Let the discretetime linear state-space model, for control synthesis, be defined by

$$
\begin{aligned}
x_{k+1} & =\Phi x_{k}+\Gamma u_{k}+\Gamma_{d} d_{k} \\
d_{k+1} & =d_{k} \\
y_{k} & =E x_{k} \\
z_{k} & =H y_{k}
\end{aligned}
$$

where $x_{k} \in \mathbb{R}^{n}$ is the state vector, $u_{k} \in \mathbb{R}^{m}$ is the control vector, $y_{k} \in \mathbb{R}^{p}$ is the vector of observed variables, $d_{k} \in \mathbb{R}^{q}$ is the vector of disturbances and $z_{k} \in \mathbb{R}^{q}(q \leq m)$, is the vector of controlled variables. The disturbance vector is included to model mismatches between the plant and the nominal model as well as the external disturbances acting on the plant. The pair $(E, \Phi)$ is assumed to be detectable with $E$ full row rank. Additionally, the disturbance vector is assumed to be estimated by a suitable observer. ${ }^{25}$

The objective of the control system is to asymptotically eliminate the tracking error given a reference command $r_{s s}$ in the presence of a disturbance $d_{k}$ and given constraints on the state and control vectors $x_{k} \in \mathbf{X}, u_{k} \in \mathbf{U}$, where $\mathbf{X}$ and $\mathbf{U}$ are closed, bounded and convex sets expressed by linear inequalities. This problem corresponds to finding a new equilibrium point for the plant in steady-state, which is essentially a determination of the steady-state target vectors $x_{s s}\left(r_{s s}, d_{k}\right)$ and $u_{s s}\left(r_{s s}, d_{k}\right)$. The constrained steady-state or target values of states and inputs are calculated in a least-squares sense, with an effort to minimize the difference of demanded $\left(r_{s s}\right)$ and reachable $\left(z_{s s}\right)$ controlled variables. The resulting constrained target calculation problem is given by

$$
\begin{gathered}
\min _{x_{s s}, u_{s s}} J\left(r_{s s}, d_{k}\right)=\left(H E x_{s s}-r_{s s}\right)^{T} Q_{s s}\left(H E x_{s s}-r_{s s}\right)+u_{s s}^{T} R_{s s} u_{s s} \\
\text { subject to: } \\
(\Phi-I) x_{s s}+\Gamma u_{s s}+\Gamma_{d} d_{k}=0 \\
u_{s s} \in \mathbf{U} \\
x_{s s} \in \mathbf{X} \\
\quad \frac{7 \text { of } 17}{\text { American Institute of Aeronautics and Astronautics }}
\end{gathered}
$$


where $Q_{s s}$ and $R_{s s}$ are weighting matrices. The second part of the cost function attempts to minimize the use of control in overactuated systems. It is important to note that the constrained problem given by Eq. (11) has $n+m$ decision variables. Moreover, its optimal solution provides constraint satisfaction only at steady state without concern for the exceedance of limitations during the transient motion. It is necessary a reparametrization of this numerical problem to reduce its computational effort, along with construction of an invariant set for the closed-loop system.

The key to the proposed reparametrization is the relation of the target state vector with the target control vector and disturbance

$$
x_{s s}=(I-\Phi)^{-1} \Gamma u_{s s}+(I-\Phi)^{-1} \Gamma_{d} d_{k}
$$

The proposed control law for the constrained tracker is based on the two-degrees-of-freedom linear quadratic regulator for the deviation system

$$
u_{k}-u_{s s}=-K_{d}\left(x_{k}-x_{s s}\right)
$$

which becomes, after the substitution of $x_{s s}$ from Eq. (12), the form

$$
u_{k}=-K_{d}\left(x_{k}-(I-\Phi)^{-1} \Gamma_{d} d_{k}\right)+\left(K_{d}(I-\Phi)^{-1} \Gamma+I\right) u_{s s}
$$

The first part of the right side of Eq. (14) feeds back to the current state vector and disturbance. The second part is the feedforward contribution of the control law, which is also responsible, as will be shown in the sequence, for ensuring the satisfaction of the input and output constraints. The feedback gain $K_{d}$ is calculated through the solution of the discrete-time Riccati equation. ${ }^{26}$ Assuming that $u_{s s}$ is constant for a given reference, it will be convenient to extend the state-space vector as $\left[\begin{array}{lll}x_{k}^{T} & d_{k}^{T} & u_{s s}^{T}\end{array}\right]^{T}$, leading to the following extended dynamical model

$$
\left[\begin{array}{c}
x_{k+1} \\
d_{k+1} \\
u_{s s}
\end{array}\right]=\left[\begin{array}{ccc}
\Phi-\Gamma K_{d} & \left(\Gamma K_{d}(I-\Phi)^{-1}+I\right) \Gamma_{d} & \Gamma\left(K_{d}(I-\Phi)^{-1} \Gamma+I\right) \\
0 & I & 0 \\
0 & 0 & I
\end{array}\right]\left[\begin{array}{c}
x_{k} \\
d_{k} \\
u_{s s}
\end{array}\right]
$$

The goal of the constrained control law is the satisfaction of the system constraints while tracking a reference value $r_{s s}$. This is achieved by computing $u_{s s}$ such as the extended state vector of Eq. (15) remains within an invariant set $O_{\infty}\left(u_{s s}\right)$. The computation of this set requires the proper formulation of the system constraints, expressed in terms of linear inequalities as

$$
\left.\left[\begin{array}{ccc}
C_{c} & 0 & 0 \\
-C_{c} & 0 & 0 \\
0 & C_{c}(I-\Phi)^{-1} \Gamma_{d} & C_{c}(I-\Phi)^{-1} \Gamma \\
0 & -C_{c}(I-\Phi)^{-1} \Gamma_{d} & -C_{c}(I-\Phi)^{-1} \Gamma \\
-K_{d} & K_{d}(I-\Phi)^{-1} \Gamma_{d} & K_{d}(I-\Phi)^{-1} \Gamma+I \\
K_{d} & -K_{d}(I-\Phi)^{-1} \Gamma_{d} & -K_{d}(I-\Phi)^{-1} \Gamma-I \\
0 & I & 0 \\
0 & -I & 0 \\
0 & 0 & I \\
0 & 0 & -I
\end{array}\right]\left[\begin{array}{c}
x_{k} \\
\\
u_{s s}
\end{array}\right] d_{k}\right] \leq\left[\begin{array}{c}
x_{\max } \\
-x_{\min } \\
x_{s s_{\max }} \\
-x_{s s_{\min }} \\
u_{\max } \\
-u_{\min } \\
d_{\max } \\
-d_{\min } \\
u_{s s_{\max }} \\
-u_{s s_{\min }}
\end{array}\right]
$$

where $x_{\max }$ and $x_{\min }, u_{\max }$ and $u_{\min }$ and $d_{\max }$ and $d_{\min }$ are the bounds on the state, control and disturbance vectors, respectively. Because of the two-degree-of-freedom nature of this controller, boundaries over state and control at steady-state can be established with enough separation to provide safety margins, which is desirable for aeronautical applications. Thus, the set $O_{\infty}\left(u_{s s}\right)$ can be constructed using the techniques described in Gilbert and Tan, ${ }^{27}$ by considering the extended dynamical system given by Eq. (15) and the constraints of Eq. (16).

From the invariant set of the extended state-space, a new set of constraints over $u_{s s}$, denoted as $\mathbf{U}_{s s_{k}}$, is computed, at each sampling time, with the slice operation $\sigma_{\left(\hat{x}_{k}, \hat{d}_{k}\right)} O_{\infty}$, where the existence of an observer 
to estimate state and disturbance values is assumed. Finally, the constrained problem Eq. (11) is redefined, after some algebra, with $u_{s s}$ as the sole vector of decision variables

$$
\begin{gathered}
\min _{u_{s s}} J\left(r_{s s}, d_{k}\right)=\left(H E x_{s s}-r_{s s}\right)^{T} Q_{s s}\left(H E x_{s s}-r_{s s}\right)+u_{s s}^{T} R_{s s} u_{s s} \\
\text { subject to: } \\
x_{s s}=(I-\Phi)^{-1} \Gamma u_{s s}+(I-\Phi)^{-1} \Gamma_{d} d_{k} \\
u_{s s} \in \mathbf{U}_{s s_{k}}
\end{gathered}
$$

It should be noted that $\mathbf{U}_{s s_{k}}$ comprises all system constraints, mapping them directly into a single polyhedral set. In addition, the complexity of the constrained target calculation is significantly reduced, with the elimination of $n$ decision variables.

\section{Application to Flexible Aircraft}

The complete set of nonlinear equations of motion with aeroelastic modes was implemented in SIMULINK environment. To provide a more realistic scenario for aeroservoelastic simulation, actuators for aerodynamic surfaces were considered with simplified first-order models. The engine dynamics was also incorporated with similar representation. Sensors models and measurement noise were taken into account in the complete nonlinear model, but they were disabled for the controller synthesis and simulation runs. Table 2 presents some physical properties of the aircraft B1 under consideration.

Table 2. B1 configuration data

\begin{tabular}{cc} 
Parameter & Value \\
\hline Mass $(\mathrm{kg})$ & 130640 \\
Wing area $\left(\mathrm{m}^{2}\right)$ & 180.80 \\
Wing span $(\mathrm{m})$ & 22.71 \\
Mean chord $(\mathrm{m})$ & 4.66 \\
$I_{x x}\left(\mathrm{~kg} \cdot \mathrm{m}^{2}\right)$ & $1.29 \times 10^{6}$ \\
$I_{y y}\left(\mathrm{~kg} \cdot \mathrm{m}^{2}\right)$ & $8.67 \times 10^{6}$ \\
$I_{z z}\left(\mathrm{~kg} \cdot \mathrm{m}^{2}\right)$ & $9.62 \times 10^{6}$ \\
$I_{x z}\left(\mathrm{~kg} \cdot \mathrm{m}^{2}\right)$ & $-7.14 \times 10^{4}$
\end{tabular}

The choice of the operation point to extract linear matrices for the controller synthesis follows the condition presented by Waszak and Schmidt, ${ }^{4}$ i.e. steady level flight at $1500 \mathrm{~m}$ of altitude and a true airspeed equals $200.4 \mathrm{~m} / \mathrm{s}$. Thus, the set of equations of motion yields a nonlinear algebraic system which can be solved for engine thrust, elevator deflection and angle of attack. For the flight condition under consideration, Table 3 shows the equilibrium values of some relevant longitudinal parameters and the first generalized flexible displacement, obtained numerically using MATLAB. Note that the aircraft experiences some small static deformations, indicated by the non-zero value of $\eta_{1}$ at steady level flight.

Table 3. Equilibrium values at $\mathrm{H}=1500 \mathrm{~m}, \mathrm{~V}=\mathbf{2 0 0 . 4} \mathrm{m} / \mathrm{s}$

\begin{tabular}{ccccc}
$\alpha(\mathrm{deg})$ & $\theta(\mathrm{deg})$ & $\eta_{1}(\mathrm{adm})$ & $\mathrm{T}(\mathrm{N})$ & $\delta_{e}(\mathrm{deg})$ \\
\hline 0.522 & 0.522 & 1.38 & 277810 & -8.24
\end{tabular}

\section{A. Velocity Vector Control System}

The basic requirement for the controller proposal and synthesis is to track values of relevant state variables and, at the same time, to reduce the oscillations caused by the aircraft flexibility. The choice of this work is to design a system capable of controlling the velocity vector, i.e. its magnitude and direction, with proper 
turn coordination by means of zeroing the lateral acceleration. Doing so, the complete closed-loop system can be demanded manually by changing the velocity vector parameters, or receiving high-level demands from some guidance system.

Any vehicle trimming trajectory can be described by the triple $(V, \gamma, \dot{\psi}) \cdot{ }^{28,29}$ Therefore, it is proposed a closed-loop closed system which tracks such variables. Figure 2 shows two separated controllers with flexible oscillation reduction capability. The longitudinal controller is responsible for tracking commanded true airspeed $V_{c}$ and flight path angle $\gamma_{c}$, while constraining the acceleration of the first flexible mode $\ddot{\eta}_{1}$, through manipulation of the elevator and engine throttle. The task of stability augmentation in the longitudinal motion is also accomplished with the constrained linear quadratic tracker technique. Similarly, the lateral controller guarantees stability augmentation and tracks commanded turn rate $\dot{\psi}_{c}$ with zero lateral acceleration, limiting the acceleration of the second flexible mode $\ddot{\eta}_{2}$. Those objectives are achieved by manipulating aileron and rudder deflection.

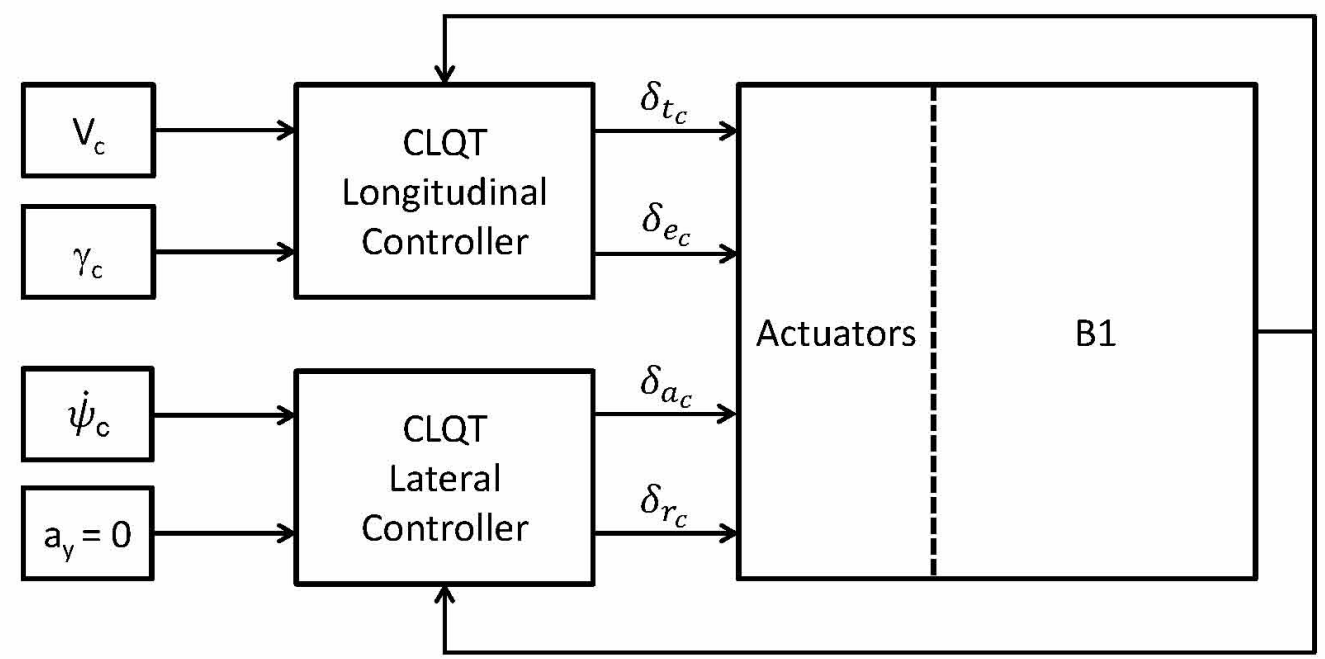

Figure 2. Velocity Vector Control System

\section{B. Longitudinal Controller Synthesis}

The state vector for longitudinal controller synthesis is given by

$$
x_{\text {long }}=\left[\begin{array}{llllllll}
V & \alpha & q & \theta & \eta_{1} & \dot{\eta}_{1} & \delta_{t} & \delta_{e}
\end{array}\right]^{T}
$$

where $V$ is the true airspeed $(\mathrm{m} / \mathrm{s}), \alpha$ is the angle of attack $(\mathrm{rad}), q$ is the pitch rate $(\mathrm{rad} / \mathrm{s}), \theta$ is the pitch angle ( $\mathrm{rad}), \eta_{1}$ is first flexible mode generalized displacement (adm), $\dot{\eta}_{1}$ is the first flexible mode generalized velocity, $\delta_{t}$ is the engine specific power $(\mathrm{adm})$ and $\delta_{e}$ is the elevator angular deflection ( $\left.\mathrm{rad}\right)$. The control vector is $u_{\text {long }}=\left[\begin{array}{ll}\delta_{t_{c}} & \delta_{e_{c}}\end{array}\right]^{T}$, which represents the engine throttle (adm) and elevator angular deflection command (rad). The simplified engine dynamics is represented by a first-order system with time constant of $0.33 \mathrm{~s}$. The elevator actuator is described also as a first-order system with time constant of $0.1 \mathrm{~s}$.

At the considered steady level flight, the open-loop longitudinal airframe eigenvalues are presented by Table 4 
Table 4. Open-loop longitudinal eigenvalues

\begin{tabular}{cccc} 
Mode & Eigenvalues & Damping & Natural freq. $(\mathrm{rad} / \mathrm{s})$ \\
\hline Phugoid & $-0.00865 \pm 0.0471 \mathrm{i}$ & 0.181 & 0.0478 \\
Short-period & $-0.580 \pm 1.51 \mathrm{i}$ & 0.358 & 1.62 \\
Flexible mode 1 & $-1.45 \pm 14.0 \mathrm{i}$ & 0.103 & 14.1
\end{tabular}

The weight matrices $Q_{d}, R_{d}$ and $N_{d}$ from the discrete-time linear quadratic cost function ${ }^{26}$ were obtained from the continuous weight matrices $Q_{l o n g}$ and $R_{\text {long }}$. The elements of the $Q_{\text {long }}$ matrix were chosen to penalize deviations of airspeed, angle of attack and pitch attitude, according to the inverse square rule, ${ }^{26}$ being expressed by

$$
Q_{\text {long }}=\left[\begin{array}{cccccccc}
1 & 0 & 0 & 0 & 0 & 0 & 0 & 0 \\
0 & (180 / \pi)^{2} & 0 & -(180 / \pi)^{2} & 0 & 0 & 0 & 0 \\
0 & 0 & 0 & 0 & 0 & 0 & 0 & 0 \\
0 & -(180 / \pi)^{2} & 0 & (180 / \pi)^{2} & 0 & 0 & 0 & 0 \\
0 & 0 & 0 & 0 & 0 & 0 & 0 & 0 \\
0 & 0 & 0 & 0 & 0 & 0 & 0 & 0 \\
0 & 0 & 0 & 0 & 0 & 0 & 0 & 0 \\
0 & 0 & 0 & 0 & 0 & 0 & 0 & 0
\end{array}\right]
$$

and the $R_{\text {long }}$ matrix was set to

$$
R_{\text {long }}=\left[\begin{array}{cc}
400 & 0 \\
0 & (90 / \pi)^{2}
\end{array}\right]
$$

The dynamical system and the weight matrices were discretized with $T_{s}=0.02 \mathrm{~s}$, and the matrices $\Phi_{l o n g}$, $\Gamma_{\text {long }}, Q_{d}, W_{d}$ and $R_{d}$ are obtained, along with the feedback gain

$$
K_{d_{\text {long }}}=\left[\begin{array}{cccccccc}
0.0191 & -0.119 & 0.00510 & 0.104 & 0 & 0 & 0.0111 & 0.0101 \\
0.0280 & 1.33 & -0.299 & -1.79 & -0.00120 & 0.0002 & 0.0159 & 0.149
\end{array}\right]
$$

The proposed longitudinal controller tracks airspeed and flight path angle, thus $z_{\text {long }}=\left[\begin{array}{ll}V & \gamma\end{array}\right]^{T}$. The target calculation problem assumes that the controlled variables are expressed in $\mathrm{m} / \mathrm{s}$ and degrees. The computation of the observer gains assumes that the variables related with the first flexible mode are not measured, thus, the observation vector comprises only the rigid-body states, along with elevator and engines states. The weights from Eq. (11) were set to $Q_{s s}=I$ and $R_{s s}=0$, since the number of controlled variables equals the number of controls. The disturbance model was chosen to have two variables and the related distribution matrix was set $\Gamma_{d}=\Gamma$. The disturbance vector was bounded between values $\pm\left[\begin{array}{ll}1 & 1\end{array}\right] \mathrm{rad}$. The observer gain $L$ was obtained by first computing $L_{x}$ and after $\bar{L}_{d}$, through the LQR technique.

\section{Lateral Controller Synthesis}

In similar fashion as the longitudinal controller, the lateral controller is synthesized using the steady level flight aeroservoelastic model, with state variables

$$
x_{\text {lat }}=\left[\begin{array}{llllllll}
\beta & p & r & \phi & \eta_{2} & \dot{\eta}_{2} & \delta_{a} & \delta_{r}
\end{array}\right]^{T}
$$

where $\beta$ is the sideslip angle (rad), $p$ is the roll rate $(\mathrm{rad} / \mathrm{s}), r$ is the yaw rate ( $\mathrm{rad} / \mathrm{s}), \phi$ is the bank angle (rad), $\eta_{2}$ is second flexible mode generalized displacement (adm), $\dot{\eta}_{2}$ is the second flexible mode generalized velocity, $\delta_{a}$ is the aileron deflection ( $\left.\mathrm{rad}\right)$ and $\delta_{r}$ is the rudder deflection (rad). The control vector is $u=\left[\begin{array}{ll}\delta_{a_{c}} & \delta_{r_{c}}\end{array}\right]^{T}$, which represents the aileron deflection command (rad) and rudder deflection command (rad). Both simplified aileron and rudder dynamics are represented by a first-order system with time constant of $0.1 \mathrm{~s}$. 
The open-loop lateral airframe eigenvalues are presented by Table 2. Note that both spiral and dutch-roll modes are unstable.

Table 5. Open-loop lateral eigenvalues

\begin{tabular}{cccc} 
Mode & Eigenvalues & Damping & Natural freq. $(\mathrm{rad} / \mathrm{s})$ \\
\hline Dutch-roll & $0.0905 \pm 1.16 \mathrm{i}$ & -0.0780 & 1.16 \\
Spiral & 0.0188 & -1.00 & 0.0188 \\
Roll subsidence & -0.620 & 1.00 & 0.620 \\
Flexible mode 2 & $-2.82 \pm 16.5 \mathrm{i}$ & 0.169 & 16.7
\end{tabular}

The lateral controller tracks turn rate and lateral acceleration, leading to $z_{l a t}=\left[\begin{array}{ll}\dot{\psi} & a_{y}\end{array}\right]^{T}$. The lateral acceleration at the center of gravity is composed by rigid-body terms and also by accelerations caused by the aircraft flexibility. At the given steady level flight condition, the lateral acceleration is given by

$$
a_{y_{C G}}=-18.5 \beta-7.62 \eta_{2}-0.128 \dot{\eta}_{2}-0.841 \delta_{a}+7.07 \delta_{r}
$$

In what follows the notation CG is dropped from $a_{y}$, where the last indicates lateral acceleration at that station. The continuous weight matrix $Q_{\text {lat }}$ penalizes variations of turn rate and lateral acceleration and $R_{\text {lat }}$ weights deviations of both lateral aerodynamic surfaces, assuming the form

$$
\begin{gathered}
Q_{\text {lat }}=\left[\begin{array}{cccccccc}
343.14 & 0 & 0 & 0 & 141.07 & 2.3803 & 15.577 & -131.05 \\
0 & 0 & 0 & 0 & 0 & 0 & 0 & 0 \\
0 & 0 & 3282.8 & 0 & 0 & 0 & 0 & 0 \\
0 & 0 & 0 & 0 & 0 & 0 & 0 & 0 \\
141.07 & 0 & 0 & 0 & 57.996 & 0.97860 & 6.4039 & -53.876 \\
2.3803 & 0 & 0 & 0 & 0.97869 & 0.01650 & 0.10810 & -0.90910 \\
15.579 & 0 & 0 & 0 & 6.4039 & 0.10810 & 0.70710 & -5.9489 \\
-131.048 & 0 & 0 & 0 & -53.876 & -0.90910 & -5.9489 & 50.049
\end{array}\right] \\
R_{\text {lat }}=\left[\begin{array}{cc}
(90 / \pi)^{2} & 0 \\
0 & (90 / \pi)^{2}
\end{array}\right]
\end{gathered}
$$

Assuming the same discretization of the continuous time system and related cost function of the longitudinal controller, the lateral feedback gain is obtained as

$$
K_{d_{\text {lat }}}=\left[\begin{array}{cccccccc}
-0.119 & -0.154 & 0.335 & -0.113 & 0.0104 & -0.00430 & 0.0933 & -0.0716 \\
-0.262 & 0.112 & -1.380 & 0.0855 & 0.00730 & 0.00200 & -0.0669 & 0.1070
\end{array}\right]
$$

It is worthy to note that there is no direct penalization of any flexible variables in the LQR cost functions of both longitudinal and lateral controllers. Finally, the observer was designed assuming $y_{\text {lat }}=$ $\left[\begin{array}{llllll}p & r & \phi & \delta_{a} & \delta_{r} & a_{y}\end{array}\right]^{T}$ and the gains $L_{x}, L_{d}$ and $L$ were obtained via LQR method.

\section{Flexible Accelerations Constraints}

The key concept of this work is the limitation of the flexible modal accelerations, diminishing the influence of flexibility in the closed-loop aircraft motion and reducing structural stress caused by such flexible modes. As showed in the last section, the linear quadratic regulator synthesis does not penalize directly the flexible variables, and the computed gains provide almost no feedback of generalized modal displacements and velocities into throttle and aerodynamic surfaces commands. Limiting the generalized modal accelerations, it is expected to attenuate the flexible variables through the proposed constrained optimal control technique.

Hence, the following constraints are imposed on the generalized modal accelerations 


$$
\begin{aligned}
-0.5 & \leq \ddot{\eta}_{1} \leq 0.5 \\
-1 & \leq \ddot{\eta}_{2} \leq 1
\end{aligned}
$$

Additional constraints are imposed on the control displacements of both controllers

$$
\begin{aligned}
-0.5 & \leq \delta_{t_{c}}-\delta_{t_{c_{\text {trim }}}} \leq 0.5 \\
-15^{\circ} & \leq \delta_{e_{c}}-\delta_{e_{c_{t r i m}}} \leq 15^{\circ} \\
-10^{\circ} & \leq \delta_{a_{c}}-\delta_{a_{c_{t r i m}}} \leq 10^{\circ} \\
-10^{\circ} & \leq \delta_{r_{c}}-\delta_{r_{c_{\text {trim }}}} \leq 10^{\circ}
\end{aligned}
$$

Therefore, invariant sets are computed for longitudinal and lateral controllers based on the established constraints and the extended closed-loop state-space formulation of Eq. (15), using the MATLAB Toolbox presented by Kvasnica. ${ }^{30}$ The computed invariant polyhedral set for longitudinal control has 40 rows and $n+m+q=12$ columns. The related set for lateral control has 65 rows and 12 columns.

\section{E. Case 1: Aggressive Change of Flight Path Angle}

Because the proposed control strategy represents one form of command governor technique, it is expected to better demonstrate its capabilities during tracking maneuvers. The demand change of controlled variables is filtered in the constrained target calculation subsystem, providing more ways to attenuate the flexible modes. Figure 3 shows a comparison with observed responses of true airspeed, flight path angle and vertical acceleration, using the constrained and unconstrained linear quadratic tracker to follow an aggressive change of flight path angle from $-3^{\circ}$ to $3^{\circ}$. Clearly, the desired attenuation is achieved by retarding the closed-loop response with less variation of vertical acceleration. The variations of generalized modal displacement and velocity were strongly reduced, as shown by Fig. 4. Almost no modal velocity is noticed, and the modal displacement is smoother than the simulated response with LQT.
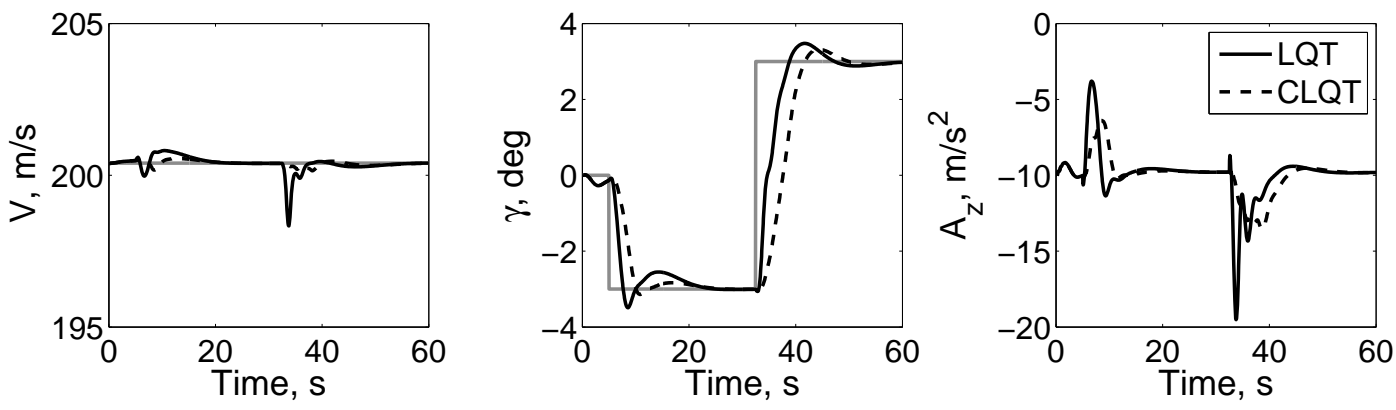

Figure 3. Evolution of the aircraft model observations in case 1

Smooth and small variations of $\eta_{1}$ and $\dot{\eta}_{1}$ are results of the constrained target calculation, which basically computes much less elevator command than LQT and retards the application of throttle, as presented by Fig. 5 .

\section{F. Case 2: Roll Reversal Maneuver}

The capability of CLQT to attenuate the anti-symmetrical aircraft flexible mode is demonstrated through a roll reversal maneuver, where the demanded turn rate changes abruptly from $+1 \mathrm{deg} / \mathrm{s}$ to $-1 \mathrm{deg} / \mathrm{s}$. Although the time responses of turn rate and bank angle do not differ significantly (Fig. 6), the lateral acceleration developed at the time of the reversal, using LQT, presents a high-frequency oscillation, which do not appear using CLQT. The evolution of $a_{y}$ with the constrained controller is smooth and free of oscillations, as presented by Fig. 7, because both $\eta_{2}$ and $\dot{\eta}_{2}$ exhibit attenuation of the high-frequency flexible motion, showed in Fig. 8 

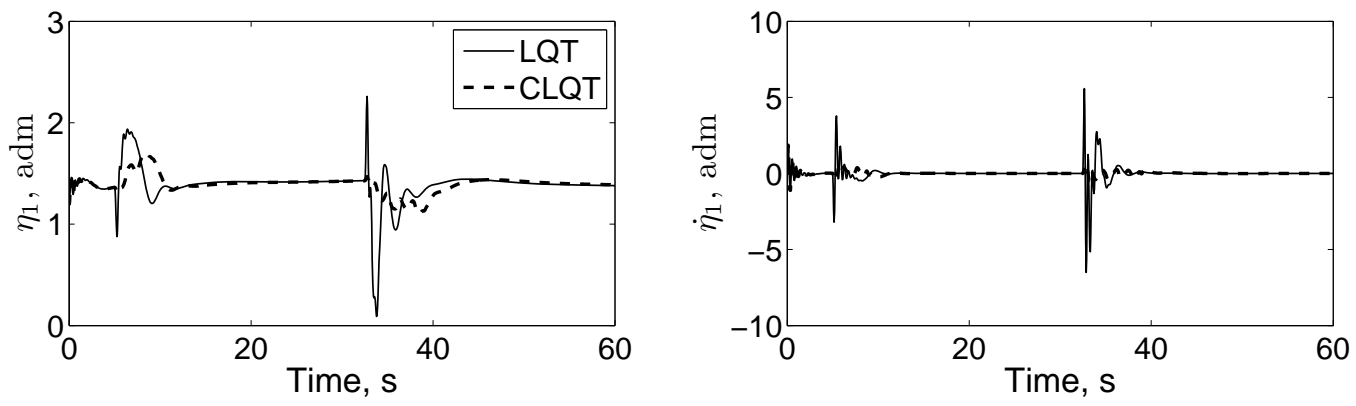

Figure 4. Evolution of the first flexible mode variables in case 1
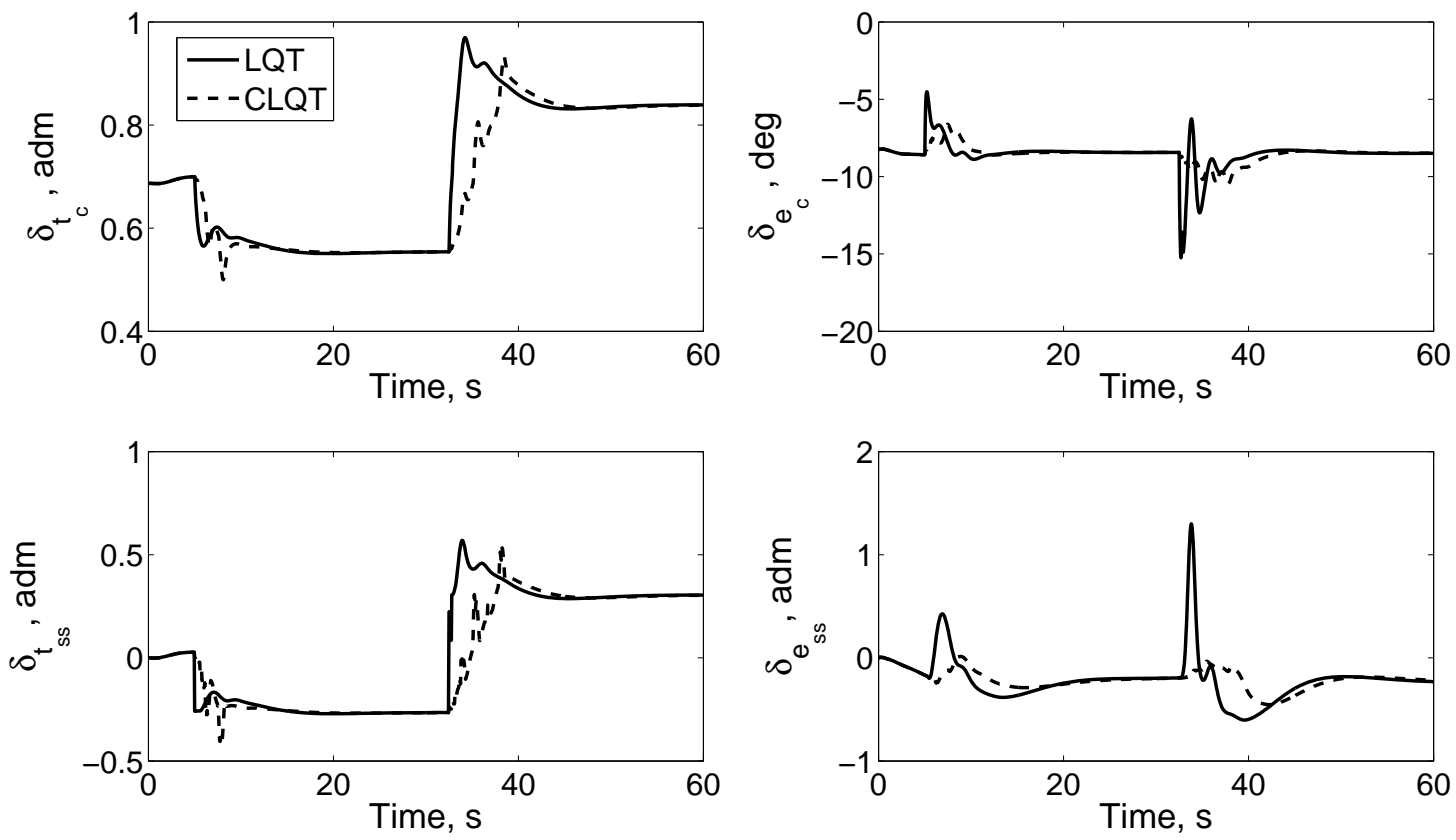

Figure 5. Evolution of the longitudinal inputs in case 1

Another benefit of this attenuation is presented by Fig. 9. Because the oscillation is almost completely eliminated, the feedback of the high-frequency motion in the aileron and rudder commands, which occurs in LQT simulation, ceases to exist using CLQT. One could argue that the attenuation should only be achieved through feedback of the flexible motion in the inputs, an approach commonly found in the literature. However, the proposed technique makes use of both feedforward (constrained) and feedback control, expanding the possibilities to deal with aircraft flexible modes.

\section{Conclusion}

With the development, theoretical and experimental characterization, and posterior validation of integrated flexible-rigid body dynamical models, it is straightforward to conceive open and closed-loop applications for elastic airplanes, as high-fidelity simulators and control systems at different hierarchical levels. Thus, not only stabilization of flexible dynamical modes is possible, but also controllers to track relevant state variables might be easier to conceive and synthesize through such integrated aeroservoelastic models.

In this work, variables related with the trajectory control of the aircraft were tracked while the flexible 

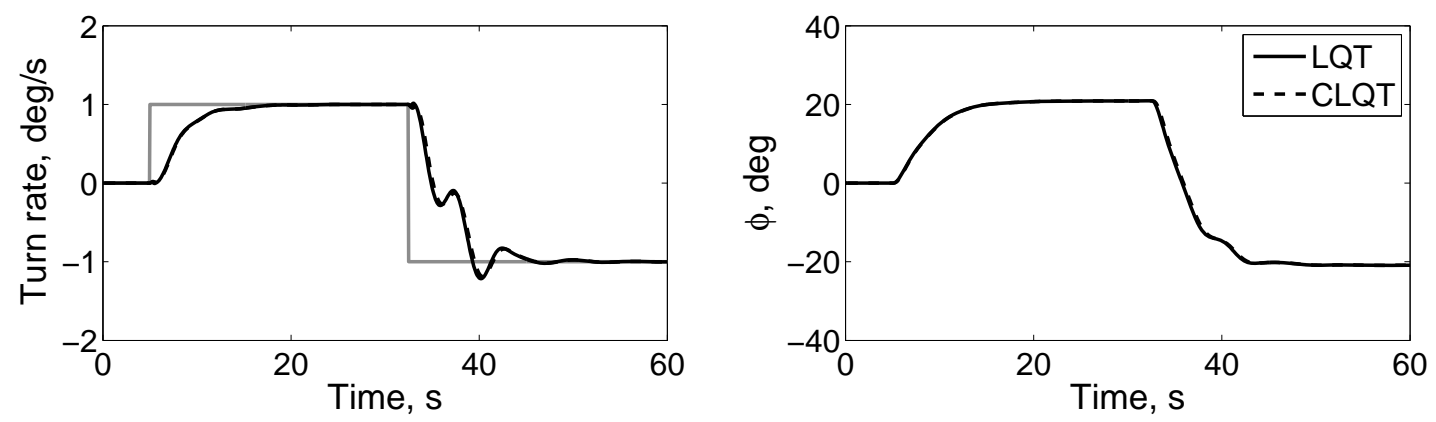

Figure 6. Evolution of the aircraft model observations in case 2

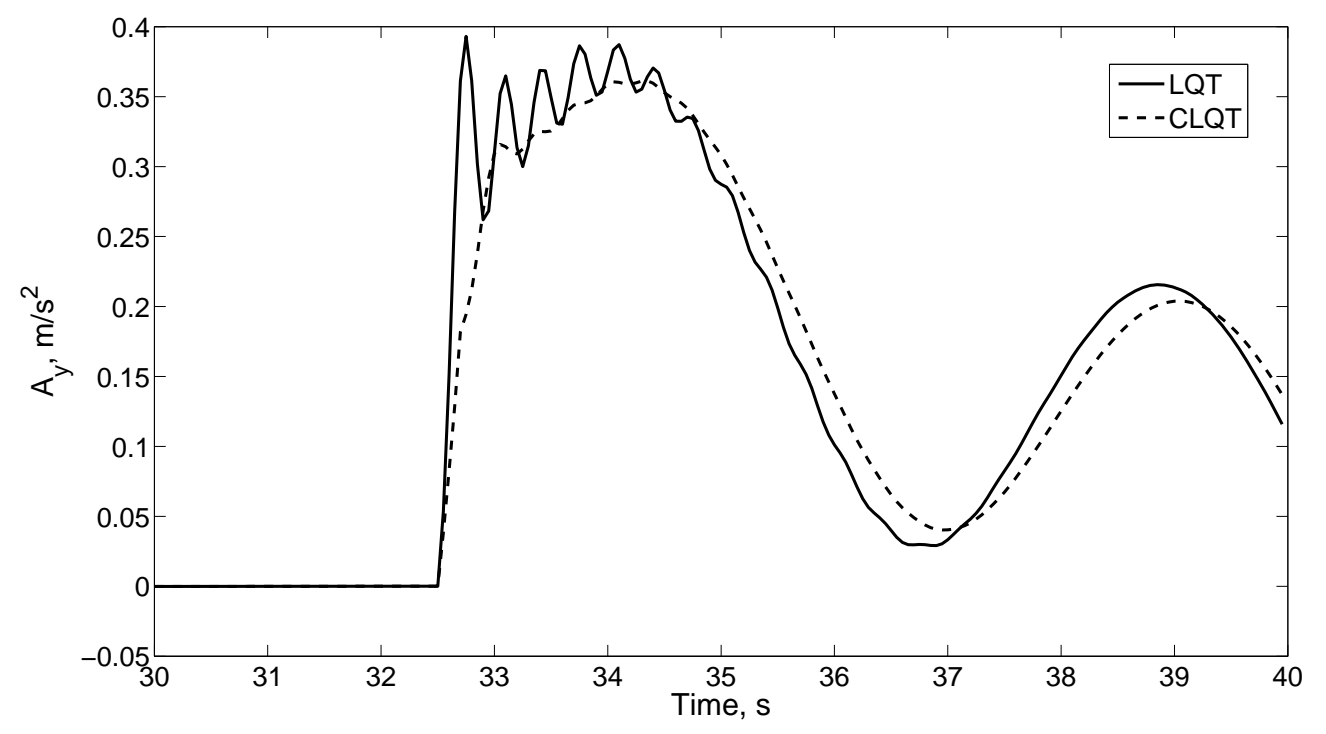

Figure 7. Evolution of the aircraft lateral acceleration in case 2

modes were considerably attenuated. The application of the constrained linear quadratic tracker turns the synthesis of such controller into a low complexity task, since the flexible variables are not taken into account in the feedback gain computation. The obtained results showed proper attenuation of the symmetric and anti-symmetric flexible modal accelerations when it applied to the B-1 bomber model. Moreover, modal displacements and velocities are also reduced when the aircraft encounters a vertical discrete gust.

For future work, it would be interesting to investigate possibilities to improve the performance of the closed-loop system, through employment of redundant aerodynamic surfaces or enlargement of the controller domain of operation using model predictive control.

\section{Acknowledgments}

This research was partially supported by the DLR, German Aerospace Center and by the DCTA, Departamento de Ciência e Tecnologia Aeroespacial, Brazil. The first author also gratefully acknowledge the financial support of FAPESP - Fundação de Amparo à Pesquisa do Estado de São Paulo - under Grant 2013/09784-4. 

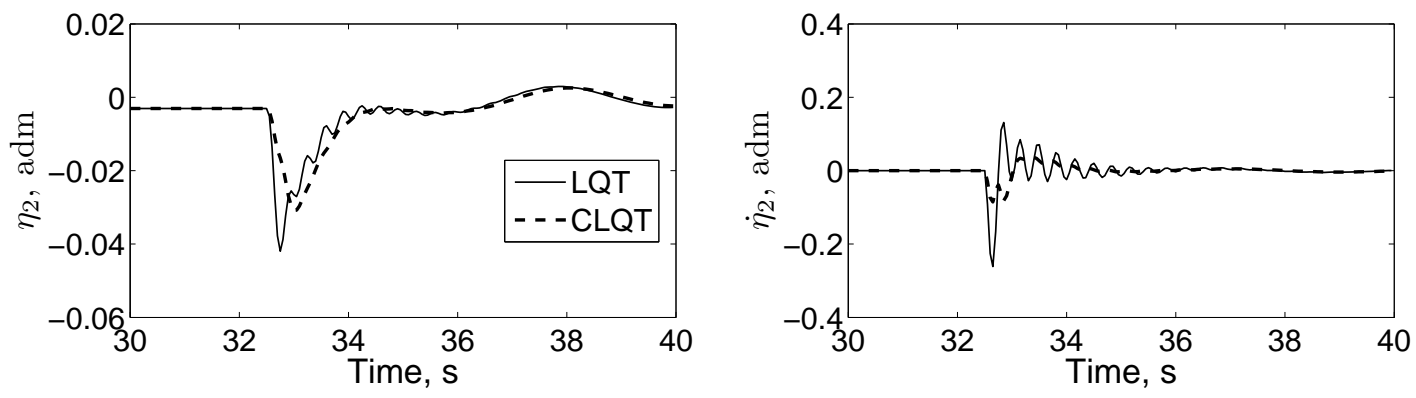

Figure 8. Evolution of the second flexible mode variables in case 2
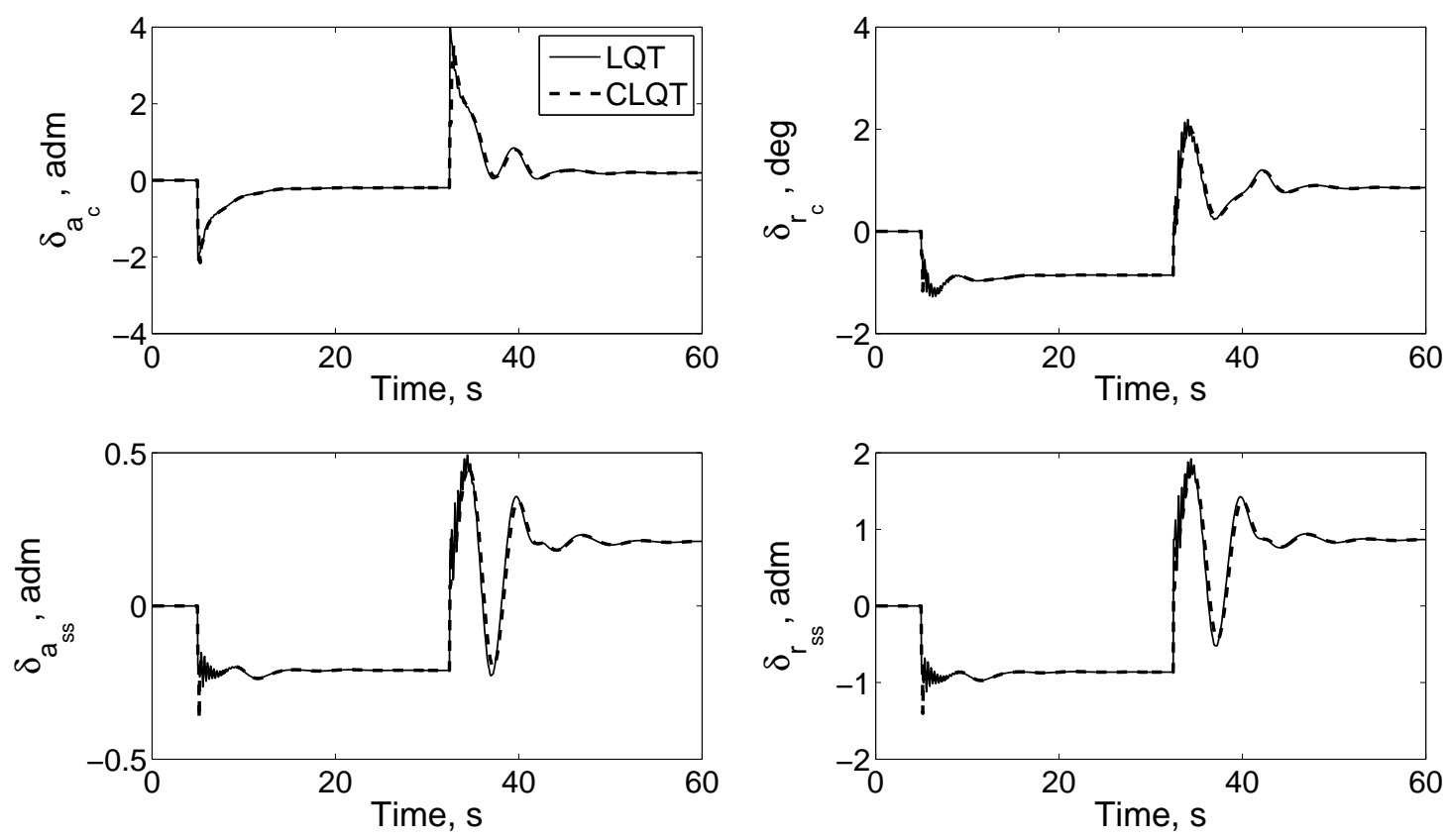

Figure 9. Evolution of the lateral-directional inputs in case 2

\section{References}

${ }^{1}$ Gates, S. and Lyon, H., "A Continuation of Longitudinal Stability and Control Analysis; Part I, General Theory," Tech. Rep. 2027, H.M.S.O., 1944.

${ }^{2}$ Duncan, W., Principles of Control and Stability of Aircraft, Cambridge University Press, Cambridge, England, 1952.

${ }^{3}$ Milne, R., "Dynamics of the Deformable Aircraft; Parts I and II," Tech. Rep. 3345, H.M.S.O., 1964.

${ }^{4}$ Waszak, M. R. and Schmidt, D. K., "Flight Dynamics of Aeroelastic Vehicles," Journal of Aircraft, Vol. 25, No. 6, 1988, pp. $563-571$.

${ }^{5}$ Buttrill, C., Zeiler, T., and Arbuckle, P., "Nonlinear Simulation of a Flexible Aircraft in Maneuvering Flight," Proceedings of the AIAA Flight Simulation Technologies Conference, No. 87-2501, Monterey, USA, 1987.

${ }^{6}$ Waszak, M., Buttrill, C., and Schmidt, D., "Modeling and Model Simplification of Aeroelastic Vehicles: An Overview," Tech. Rep. NASA-TM-107691, 1992.

${ }^{7}$ Fornasier, L., Rieger, H., Trernel, U., and van der Weide, E., "Time-Dependent Aeroelastic Simulation of Rapid Manoeuvering Aircraft," Proceedings of the AIAA Aerospace Sciences Meeting \& Exhibit, No. 2002-0949, Reno, USA, 2002.

${ }^{8}$ Garcia, J. A., "Numerical Investigation of Nonlinear Aeroelastic Effects on Flexible High-Aspect-Ratio Wings," Journal of Aircraft, Vol. 42, No. 4, 2005, pp. 1025-1036.

${ }^{9}$ Schütte, A., Einarsson, G., Raichle, A., Schöning, B., Mönnich, W., Orlt, M., Neumann, J., Arnold, J., and Forkert, T., "Numerical Simulation of Maneuvering Aircraft by Aerodynamic, Flight-Mechanics, and Structural-Mechanics Coupling," Journal of Aircraft, Vol. 46, No. 1, 2005, pp. 53-64. 
${ }^{10}$ Cavagna, L., Masarati, P., and Quaranta, G., "Simulation of Maneuvering Flexible Aircraft by Coupled Multibody/CFD," Proceedings of the Multibody Dynamics, ECCOMAS Thematic Conference, Warsaw, Poland, 2009.

${ }^{11}$ Garrec, C. L., Humbert, M., and Lacabanne, M., "Identification of the Aeroelastic Model of a Large Transport Civil Aircraft for Control Law Design and Validation," Proceedings of the 22nd Congress of International Council of the Aeronautical Sciences - ICAS, Harrogate, UK, 2000.

${ }^{12}$ Theodore, C. R., Ivler, C. M., Tischler, M. B., Field, E. J., Neville, R. L., and Ross, H. P., "System Identification of Large Flexible Transport Aircraft," Proceedings of the AIAA Atmospheric Flight Mechanics Conference, No. 2008-6894, Honolulu, USA, 2008

${ }^{13}$ de Oliveira Silva, B. G., "Data Gathering and Preliminary Results of the System Identification of a Flexible Aircraft Model," Proceedings of the AIAA Atmospheric Flight Mechanics Conference, No. 2011-6355, Portland, USA, 2011.

${ }^{14}$ de Oliveira Silva, B. G. and Mönnich, W., "System Identification of Flexible Aircraft In Time Domain," Proceedings of the AIAA Atmospheric Flight Mechanics Conference, No. 2012-4412, Minneapolis, USA, 2012.

${ }^{15}$ Shearer, C. M. and Cesnik, C. E. S., "Trajectory Control for Very Flexible Aircraft," Journal of Guidance, Control, and Dynamics, Vol. 31, No. 2, Mar.-Apr. 2008, pp. 340-357.

${ }^{16}$ Silvestre, F. J. and Paglione, P., "Dynamics and Control of a Flexible Aircraft," Proceedings of the AIAA Guidance, Navigation, and Control Conference and Exhibit, No. AIAA-2008-6876, Honolulu, USA, Aug. 2008.

${ }^{17}$ Wildschek, A., Maier, R., Hoffmann, F., Jeanneau, M., and Baier, H., "Active Wing Load Alleviation with an Adaptive Feed-forward Control Algorithm," Proceedings of the AIAA Guidance, Navigation, and Control Conference and Exhibit, No. AIAA-2006-6054, Keystone, USA, Aug. 2006.

${ }^{18}$ Wildschek, A., Maier, R., Hahn, K. U., Leißling, D., Preß, M., and Zach, A., "Flight Test with an Adaptive Feed-Forward Controller for Alleviation of Turbulence Excited Wing Bending Vibrations," Proceedings of the AIAA Guidance, Navigation, and Control Conference and Exhibit, No. AIAA-2009-6118, Chicago, USA, Aug. 2009.

${ }^{19}$ Haghighat, S., Liu, H. H., and Martins, J. R., "Application of Model Predictive Control to Gust Load Alleviation Systems," Proceedings of the AIAA Guidance, Navigation, and Control Conference and Exhibit, No. AIAA-2009-5929, Chicago, USA, Aug. 2009.

${ }^{20}$ Haghighat, S., Liu, H. H., and Martins, J. R., "Application of Robust Control Design Techniques to the Aeroservoelastic Design Optimization of a Very Flexible UAV Wing," Proceedings of the 13th AIAA/ISSMO Multidisciplinary Analysis Optimization Conference, No. AIAA-2010-9123, Fort Worth, USA, Sep. 2010.

${ }^{21}$ Haghighat, S., Liu, H. H., and Martins, J. R., "Mixed-Norm Multi-Objective Robust Controller Applied to a Very Flexible Aircraft," Proceedings of the AIAA Guidance, Navigation, and Control Conference and Exhibit, No. AIAA-2011-6256, Portland, USA, Aug. 2011.

${ }^{22}$ Frost, S. A., Taylor, B. R., Jutte, C. V., Burken, J. J., Trinh, K. V., and Bodson, M., "A Framework for Optimal Control Allocation with Structural Load Constraints," Proceedings of the AIAA Guidance, Navigation, and Control Conference and Exhibit, No. AIAA-2011-8112, Portland, USA, Aug. 2011.

${ }^{23}$ de Almeida, F. A., Guerra, E. B., d'Oliveira, F. A., and Mello, A. W., "Constrained Linear Quadratic Tracker for Optimal Flight Performance," Journal of Guidance, Control, and Dynamics, Vol. 35, No. 6, 2012, pp. 1911-1918.

${ }^{24}$ Waszak, M., Davidson, J., and Schmidt, D., "A Simulation Study of the Flight Dynamics of Elastic Aircraft; Vol. I and II," Tech. Rep. NASA-CR-4102, 1987.

${ }^{25}$ Maeder, U., Borrelli, F., and Morari, M., "Linear Offset-free Model Predictive Control," Automatica, Vol. 45, No. 10, Oct. 2009, pp. 2214-2222.

${ }^{26}$ Bryson, A., Control of Spacecraft and Aircraft, Princeton University Press, Princeton, NJ, 1994, Appendix D.

${ }^{27}$ Gilbert, E. and Tan, K., "Linear Systems with State and Control Constraints: The Theory and Application of Maximal Output Admissible Sets," IEEE Transactions on Automatic Control, Vol. 36, No. 9, 1991, pp. 1008-1020.

${ }^{28}$ Kaminer, I., Pascoal, A., Hallberg, E., and Silvestre, C., "Trajectory Tracking for Autonomous Vehicles: An Integrated Approach to Guidance and Control," Journal of Guidance, Control, and Dynamics, Vol. 21, No. 1, Jan.-Feb. 1998, pp. 29-38.

${ }^{29}$ Goman, M., Khramtsovsky, A., and Kolesnikov, E., "Evaluation of Aircraft Performance and Maneuverability by Computation of Attainable Equilibrium Sets," Journal of Guidance, Control, and Dynamics, Vol. 31, No. 2, 2008 , pp. 329-339.

${ }^{30}$ Kvasnica, M., Real-Time Model Predictive Control via Multi-Parametric Programming: Theory and Tools, VDM, Saarbrücken, Germany, 1st ed., 2009, Chap. 1. 